\title{
Erratum to: Genetic Counselors' Experiences Regarding Communication of Reproductive Risks with Autosomal Recessive Conditions found on Cancer Panels
}

\author{
Sarah Mets ${ }^{1} \cdot$ Rebecca Tryon ${ }^{2} \cdot$ Patricia McCarthy Veach $^{3} \cdot$ Heather A. Zierhut ${ }^{1}$
}

Published online: 1 December 2015

(C) National Society of Genetic Counselors, Inc. 2015

Erratum to: J Genet Counsel

DOI 10.1007/s10897-015-9892-y

We would like to note the following corrections:

- Abstract - confusion with 189 versus 187 responses

- The survey assessed 189 cancer genetic counselors' experiences discussing reproductive risks with patients at risk to carry a mutation or variant of uncertain significance (VUS) in a gene associated with both an autosomal dominant cancer risk and an autosomal recessive syndrome.
To clarify, 189 met inclusion criteria, but only 187 continued with the survey.

- Page 3

- If a known carrier of an ATM mutation had a child with a partner of unknown carrier status, the chance for them to have a child with ataxia telangiectasia would be 1/800.

This should read $1 / 400$, not $1 / 800$

The authors regret these errors.

The online version of the original article can be found at http://dx.doi.org/ 10.1007/s10897-015-9892-y.

Heather A. Zierhut zier0034@umn.edu

1 Department of Genetics, Cell Biology, \& Development, University of Minnesota, 321 Church Street, 6-160 Jackson Hall,

Minneapolis, MN 55455, USA

2 Fairview Health Services, Minneapolis, MN 55455, USA

3 Department of Educational Psychology, University of Minnesota, Minneapolis, MN 55455, USA 